\title{
Evaluation of implant fit and frontal plane alignment after bi-compartmental knee arthroplasty using patient-specific instruments and implants
}

\author{
Joerg Arnholdt ${ }^{1}$, Yama Kamawal ${ }^{1}$, Boris Michael Holzapfel ${ }^{1}$, Axel Ripp ${ }^{2}$, Maximilian Rudert ${ }^{1}$, \\ Andre Friedrich Steinert ${ }^{1,3}$
}

\begin{abstract}
${ }^{1}$ Department of Orthopaedic Surgery, König-Ludwig-Haus, Julius-MaximiliansUniversity, Würzburg, Germany

${ }^{2}$ Department of Trauma and Orthopaedic Surgery, Elblandkliniken, Radebeul, Germany ${ }^{3}$ Department of Trauma and Orthopaedic Surgery, Agatharied Hospital, Hausham, Germany
\end{abstract}

Submitted: 6 June 2017

Accepted: 6 September 2017

Arch Med Sci 2018; 14, 6: 1424-1431

DOI: https://doi.org/10.5114/aoms.2018.79007

Copyright (c) 2018 Termedia \& Banach

\begin{abstract}
Introduction: The goals of successful bi-compartmental knee arthroplasty are to achieve correct fit and positioning of the implant, while appropriately correcting the mechanical alignment of the leg after surgery. As these requirements are not always reliably fulfilled using off-the-shelf implant systems, newer approaches for bi-compartmental resurfacing have been explored.

Material and methods: In this article we report the radiographic results of 30 patients with anteromedial osteoarthritis (OA) who were treated with a novel patient-specific fixed-bearing bi-compartmental knee resurfacing system using custom-made implants and instruments. Utilizing standardized pre- and postoperative radiographic analyses (based on anterior-posterior and lateral, anterior-posterior weight-bearing full-length radiographs, patella skyline views and preoperative computed tomography (CT) scanning) implant fit and positioning as well as correction of the mechanical axis (hipknee-ankle angle, HKA) were determined.

Results: On average, HKA was corrected from $173.4 \pm 3.47^{\circ}$ preoperatively to $179.4 \pm 2.85^{\circ}$ postoperatively. The coronal femoro-tibial angle was corrected on average $5.61^{\circ}$. The preoperative tibial slope measured on lateral views was $6.38 \pm 2.4^{\circ}$, while the average slope in the CT-based planning protocol (iView) was $6.14 \pm 2.40^{\circ}$. Postoperative lateral tibial slope was determined to be $5.77 \pm 1.97^{\circ}$. The thickness of the posterior femoral cuts was measured intraoperatively and, in all cases, corresponded well to the targeted thickness of the cuts provided by the iView. The joint line was preserved in all cases and the average Insall-Salvati index was $1.078 \pm 0.11$ pre- and 1.072 \pm 0.11 postoperatively. The fit of the implant components measured by overor underhang was excellent throughout $(<1.01 \mathrm{~mm})$.

Conclusions: Custom-made bicompartmental knee arthroplasty can ensure optimized fitting and positioning of the implant with restoration of the leg axis. These implants could be considered as an alternative primary solution for knee surgeons treating bi-compartmental disease.
\end{abstract}

Key words: bi-compartmental, knee arthroplasty, patient-specific, knee alignment, knee osteoarthritis, implant positioning.

\author{
Corresponding author: \\ Joerg Arnholdt MD \\ Department \\ of Orthopaedic Surgery \\ König-Ludwig-Haus \\ Julius-Maximilians- \\ University \\ Brettreichstraße 11 \\ 97074 Würzburg, Germany \\ Phone: +49 9318033124 \\ E-mail: j-arnholdt.klh@ \\ uni-wuerzburg.de
}




\section{Introduction}

Symptomatic bi-compartmental osteoarthritis (OA) of the knee remains a therapeutic challenge for orthopedic surgeons. A recent study has shown that up to $28 \%$ of the patients planned for primary total knee arthroplasty (TKA) exhibit only bi-compartmental osteoarthritis that is present in either the medial or lateral compartments in addition to the patello-femoral joint [1]. This finding has been corroborated by another study, which utilized preoperative magnetic resonance imaging (MRI) analyses of osteoarthritic changes, to confirm that up to $36 \%$ of patients would be eligible for bi-compartmental knee arthroplasty (BKA) instead of being treated by TKA [2].

Despite the experiences and advances that have been made possible with total knee arthroplasty, there is a considerable proportion of patients (around 20\%) after primary TKA, who are still suffering from discomfort or pain despite or because of their operation [3, 4]. In younger patients (under 55 years), overall satisfaction seems to be better after unicondylar knee arthroplasty (UKA) as compared to TKA [5]. However, there are many patients who suffer from symptomatic bi-compartmental osteoarthritis. For these patients the implantation of a UKA would mean under-treatment, whereas TKA would mean overtreatment with the necessity of sacrificing an otherwise healthy knee compartment along with the anterior cruciate ligament, which is well known to facilitate natural knee proprioception and kinematics $[6,7]$. For these patients a bone and cruciate ligament-sparing resurfacing technique with preservation of natural knee kinematics would be desirable. For this purpose, BKA has been introduced to the clinical arena using off-the-shelf modular (separate uni-compartmental and patello-femoral replacement components) or monoblock femoral components [8]. So far, the use of these off-the-shelf BKA systems has resulted in good functional outcomes in the majority of the patients [8], but also high complication rates of up to $18-30 \%$ in the populations examined with high revision rates and a rate of conversion to TKA of up to $18 \%[8,9]$. Primary factors for revision are pain, malalignment or instability, which is attributed to the technical complexity and insufficient fit of the implant to the patients' individual anatomy [9]. To overcome these limitations, a patient-specific BKA system (iDuo; ConforMIS Inc.) has been introduced utilizing custom made individual instruments and implants, that are fabricated to match patient anatomy on the basis of computed tomography (CT) scans using computer-aided design and manufacturing (CAD/CAM) technologies [10-12]. This technology has already been introduced into the clinic for UKA [13], pro- viding excellent radiological [14] and initial clinical outcomes $[15,16]$. The patient-specific BKA is designed with a bone-sparing technique that covers the cortical bone of the diseased distal femur and trochlea while preserving the cruciate ligaments as well as the intact tibio-femoral compartment [10-12].

The aim of this first retrospective radiologic analysis was to evaluate the precision of implant positioning and correction of leg alignment in patients with anteromedial OA who have been treated with the customized BKA. We hypothesized that the patient-specific BKA solution would result in a precise component fit and accurate leg axis correction.

\section{Material and methods}

This retrospective study was performed in accordance with the Declaration of Helsinki, and was approved by the institutional review board (IRB) of the University of Wuerzburg (No. 2016091601).

All the customized BKAs (medial compartment plus patellofemoral compartment; 16 right, 14 left) were performed using a patient-specific cruciate retaining knee resurfacing system (iDuo; ConforMIS, Inc; Burlington, MA, USA) between 2011 and 2013. This system is CE Mark approved and cleared by the United States Food and Drug Administration (FDA). The average age of the patients (14 women and 16 men) at the time of surgery was $61.2 \pm 7.84$ years (50-82 years). Greater than $15^{\circ}$ (valgus, varus, flexion) of deformity and ligamentous instability were contraindications for this surgery. All procedures were performed by two experienced senior surgeons.

Preoperative computed tomography of the affected leg was performed by scanning the knee, the femoral head and talus center according to a standard protocol (http://www.conformis.com/ healthcare-professionals/imaging-professionals) as described previously [10-12]. The patient-specific BKA system (which is available for resurfacing of the anteromedial as well as the anterolateral compartment) is designed using a software algorithm (iFit Technology) that registers the articular surfaces of the knee, the areas of disease and osteophyte formation, correction of any malalignment, and generates a BKA design that precisely matches the patient's anatomy. Thereafter, the patient-specific implant components and instruments are manufactured using 3D rapid prototyping technology. A representative surgical planning protocol (iView 2.0) is shown in Figure 1, comprising four femoral (upper panels) and four tibial (lower panels) planning images. In this protocol, any osteophytes that interfere with correct positioning of the instruments or implants are marked in red and have to be removed before positioning 


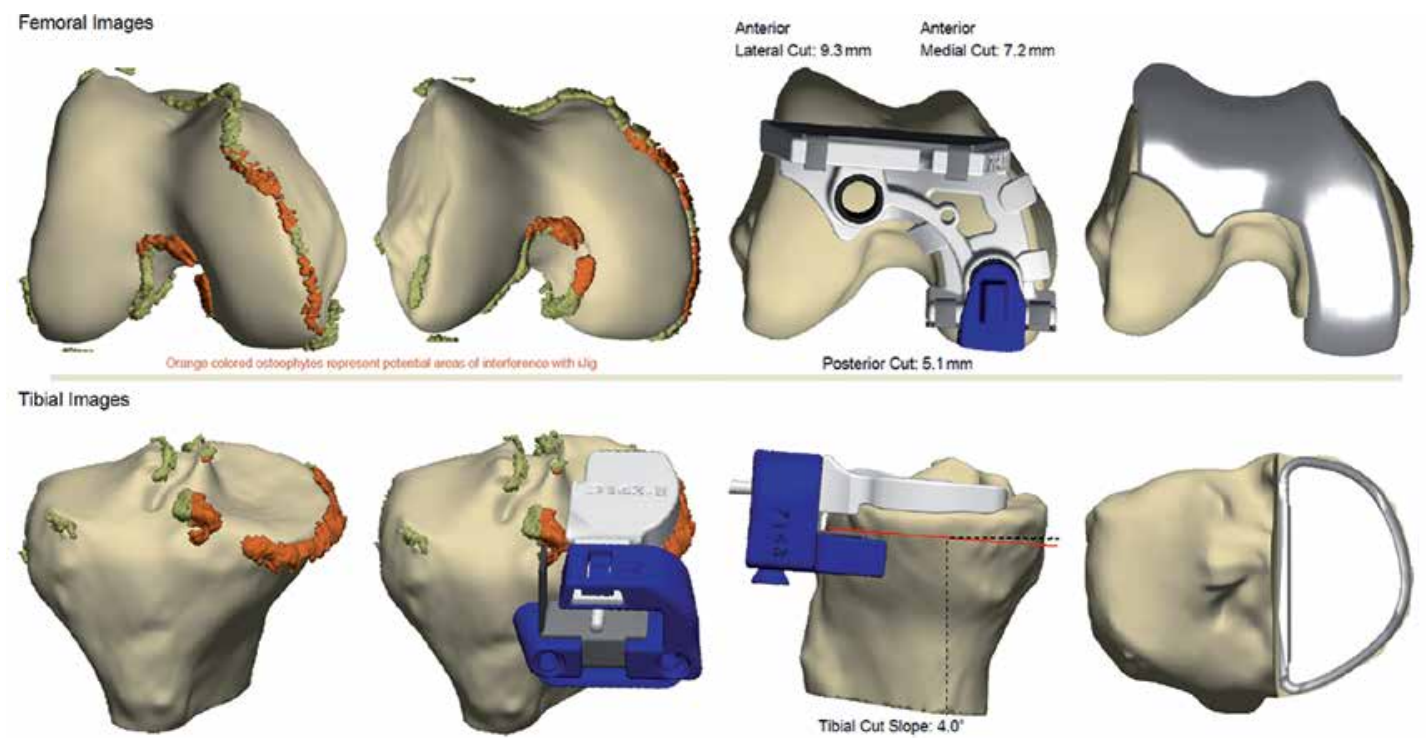

Figure 1. Representative surgical plan (iView 2.0) for patient-specific bicompartmental knee replacement

of the instruments. All further osteophytes are labeled green (Figure 1; left panels). With this patient-specific conformity to the cortical femoral bone it is possible to generate a femoral component that truly resurfaces the anteromedial compartment while requiring only cartilage removal distally, in addition to anterior and posterior bone cuts that are facilitated by the femoral jig instrumentation (Figure 1; upper panels on the right). The respective thicknesses of targeted bone cuts anterior medial or lateral as well as posterior cuts - are also provided (Figure 1; upper right panels). The tibial bone cut is facilitated by the tibial jig instrumentation (which aims to restore the natural tibial slope) (Figure 1; lower panels). All essential information about knee geometry, mechanical and anatomical axes as well as projected cutting planes are integrated in the patient-specific jigs. The femoral and tibial implants are designed to feature exact rim coverage of the respective cortical bone while matching the articular surfaces (Figure 1; right panels).

\section{Surgical procedure}

After a midline skin incision, a medial parapatellar arthrotomy is performed and the linea terminalis of the intact compartment is labeled. Then, the medial meniscus is excised and all interfering osteophytes (Figure 1, labeled in red) are resected. Using a curette all remaining cartilage is removed in both diseased compartments up to the linea terminalis. The tibial navigation balancing chips are then tested while the knee is flexed to $20^{\circ}$. The chips are provided in four different thicknesses ( $1 \mathrm{~mm}$ increments $A-D)$ for extension gap balancing. This enables proper ligament balancing until the ideal tension is determined $(1 \mathrm{~mm}$ medi- al and 1 to $2 \mathrm{~mm}$ lateral joint space opening). The tibial ijig cutting instrument is placed onto the appropriate navigation chip. Then, alignment is confirmed using an external alignment guide, and the tibial ijig is fixed with two or three tibial pins prior to completion of the sagittal and horizontal tibial cuts. The morphology and height of the resected tibial bone can be correlated to the morphology and height given in the iView planning protocol for internal quality control.

Thereafter, the flexion gap is balanced using an 8 or $10 \mathrm{~mm}$ balancing chip and the femoral ijig instrument together with an L-guide is mounted onto the femoral condyle. Two peg holes are then drilled and pinned. The L-guide, whose thickness corresponds to the intended posterior condyle resection, is removed and the posterior femoral cut is performed. The correct thickness of the resected bone piece should be confirmed with the iView before the controlled anterior femoral cut is made (Figure 1; upper panels). Several inter-digitization holes are drilled with a $2 \mathrm{~mm}$ drill on the femoral side for better cement penetration. The tibial preparation is completed by confirming the fit of the tibial template, drilling of two peg holes and insertion of a keel punch. Trial components can be used for final control of implant fit and ligament tension. Thereafter, components are cemented using a standard technique before the original polyethylene liner is inserted. The detailed surgical procedure has been described previously $[11,12]$.

\section{Radiographic analyses}

Radiographic analyses were performed prior to surgery and one week after surgery with a strictly antero-posterior (AP) view, a lateral view (including a referencing sphere) and a skyline view. Ad- 
ditionally, AP weight bearing long leg radiographs were performed in full extension. Tibial component fit was evaluated on postoperative AP and lateral views: An accurate fitting of the prosthetic components was targeted, with discrimination between a perfect fit $( \pm 2 \mathrm{~mm})$, overhang or subsidence [17]. The tibial slope was measured on preoperative lateral views and in the iView planning protocol. Measured values were compared to the values measured on postoperative lateral views. The aim of surgery was to restore the patient-specific anatomic slope as indicated by the iView planning protocol. Alterations of the patella height pre- and postoperatively, as an indicator for any joint line alterations, were determined on lateral views (Figure 2) using the Insall-Salvati ratio and the modified Insall-Salvati ratio [18]. The lateral patella tilt was measured on skyline views $[19,20]$. Pre- and postoperative deviation from the ideal mechanical axis was determined using pre- and postoperative AP weight bearing long leg radiographs. Within this context, the hip-knee-ankle angle (HKA), which is the angle between the mechanical axis of the femur (FMA) and the mechanical axis of the tibia (TMA), was determined, with both lines crossing at the center of the knee (Figure 3). The FMA constitutes the connection between the center of the femoral head and the center of the knee, while the TMA connects the knee center with the center of the ankle. The ideal HKA was set at $180 \pm 3^{\circ}$ varus/valgus. All measurements were performed twice by three independent reviewers (orthopedic surgeons: JA, YK, $\mathrm{BMH})$.

\section{Statistical analysis}

For the descriptive analyses (mean \pm standard deviation) Microsoft Excel 2007 (Microsoft Corporation, Redmond, USA) was used. Normal distribution (Gaussian distribution) of the collected data was confirmed using the Kolmogorov-Smirnov test. A paired $t$-test (SPSS, IBM, Germany) was used to compare values of means for determined

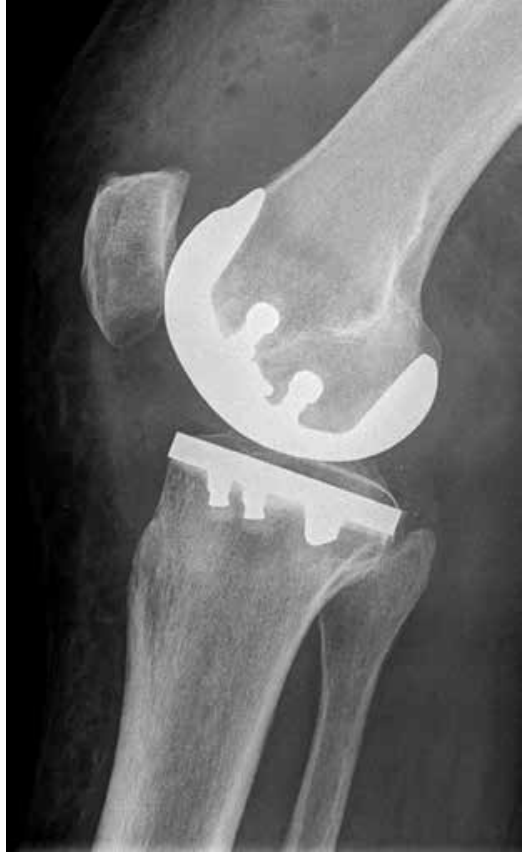

Figure 2. Lateral postoperative radiographic view of a representative knee joint after iDuo implantation

parameters pre- and postoperatively, and a level of $p<0.05$ was considered statistically significant.

\section{Results}

\section{Implant positioning}

An ideal fit of the tibial component was registered in 19 patients without any measurable overhang or underhang on AP or lateral view radiographs. A minor lateral overhang $(<2 \mathrm{~mm})$ of the tibial component was recorded in 7 cases $(1.01$ $\pm 0.46 \mathrm{~mm})$ and a minor underhang $(<2 \mathrm{~mm})$ of the tibial plateau in 4 patients $(0.87 \pm 0.47 \mathrm{~mm})$. None of the patients had an overhang/underhang of $3 \mathrm{~mm}$ or more in any direction. Overall, representative lateral radiographic views showed an excellent fit of the femoral and tibial components without any signs of significant overhang, underhang or femoral notching (Figure 2).

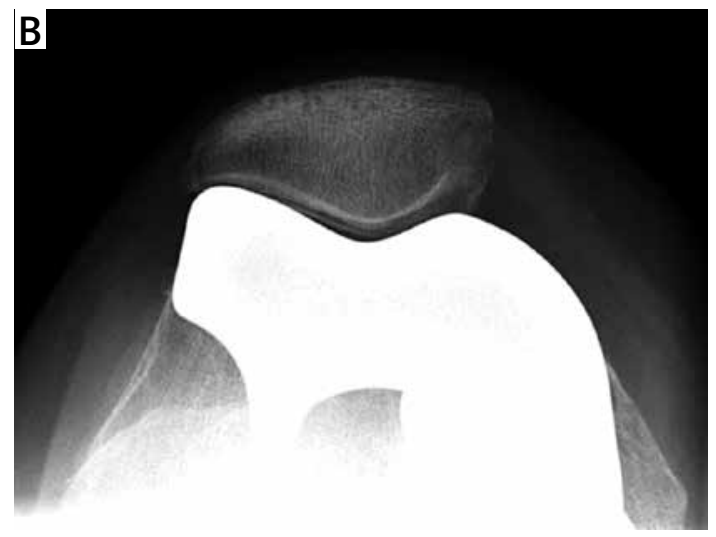

Figure 3. Skyline view of the knee joint before and after iDuo implantation 
The slope of the tibial component was intended to restore the natural slope of the tibial plateau. Preoperatively, the mean tibial slope measured on lateral view X-rays was $6.38 \pm 2.4^{\circ}$, and $6.14 \pm 2.40^{\circ}$ in the iView planned protocol. Postoperatively, this was consistently reproduced, resulting in a mean slope of the tibial implant of $5.77 \pm 1.97^{\circ}$ upon determination on postoperative lateral view $\mathrm{X}$-rays (Figure 2).

The patella height was well preserved as demonstrated by the values of the Insall-Salvati ratio. Pre-operatively the average Insall-Salvati ratio was $1.078 \pm 0.11$, while the corresponding post-operative measurement was $1.072 \pm 0.11$. This was also confirmed by the modified Insall-Salvati ratio, which was $1.747 \pm 0.28$ pre- and $1.733 \pm 0.27$ postoperatively.

Furthermore, analysis of the skyline view radiographs showed central patella tracking in all 30 cases postoperatively, with no pathologic lateral patella tilt in any of the cases (Figure 3 ).

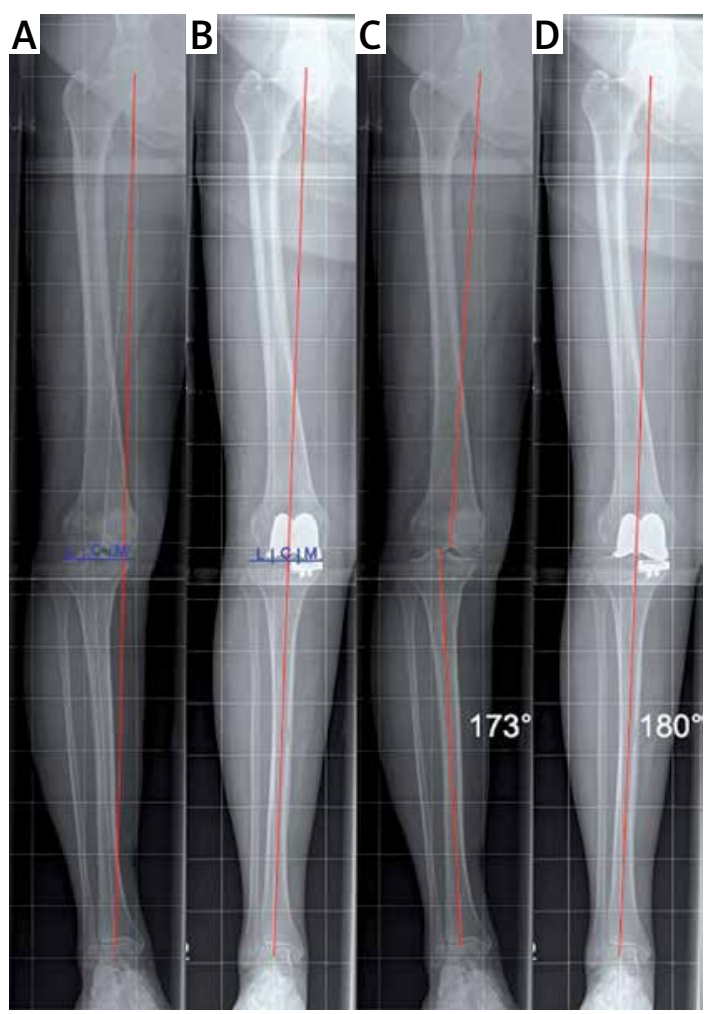

Figure 4. The hip-knee-ankle angle (HKA) was determined as the angle between the mechanical axis of the femur and the mechanical axis of the tibia (red lines), and was determined in this case $173^{\circ}$ preoperatively (A), and $180^{\circ}$ postoperatively (B). To determine the zone of the mechanical axis, the tibial plate was divided into three equal zones (lateral $=\mathrm{L}$, central $=\mathrm{C}$, medial $=\mathrm{M}$ ) and the mechanical axis (red line) that passes the tibial plate was defined according to which zone it passes through, namely medial preoperatively (C) and central postoperatively (D)
The bony thickness of the resected posterior condyle correlated exactly with the preoperatively planned resection values given by the respective iView planning protocol. With respect to posterior condyle resection, accurate matching of the actually resected bone with the plan was targeted, as this value corresponds to the posterior femoral component implant thickness as indicated on the iView (Figure 1; upper panels). In all patients, an exact match of the bony height of the resected posterior condyle could be achieved, confirming accuracy of the bone cuts resulting from the patient-specific guides.

\section{Frontal plane alignment}

Radiographic analysis of all 30 patients revealed that the HKA was corrected from 173.4 $\pm 3.47^{\circ}$ preoperatively to $179.4 \pm 2.85^{\circ}$ postoperatively (Figures 4 and 5). The range of the post-operative HKA in the patient population, the $25-75 \%$ interval, and the maximum and minimum values are illustrated in Figure 5. To determine the zone of the mechanical axis (ZMA), the tibial plate was divided into three equal zones (lateral $=\mathrm{L}$, central $=C$, medial $=M$ ) and the mechanical axis that passes the tibial plate was defined according to which zone it passes through (Figure 4). The ZMA was improved from $20.0 \%$ of the knees preoperatively crossing in the central third of the tibial plateau to over $80.0 \%$ postoperatively after bi-compartmental knee resurfacing.

No intra-operative complications were encountered in any patient. There was no need for an intraoperative system change or a modification of the patient specific instruments, as all the individual cutting blocks, drill guides and implants matched the anatomy of the individual knee joint.

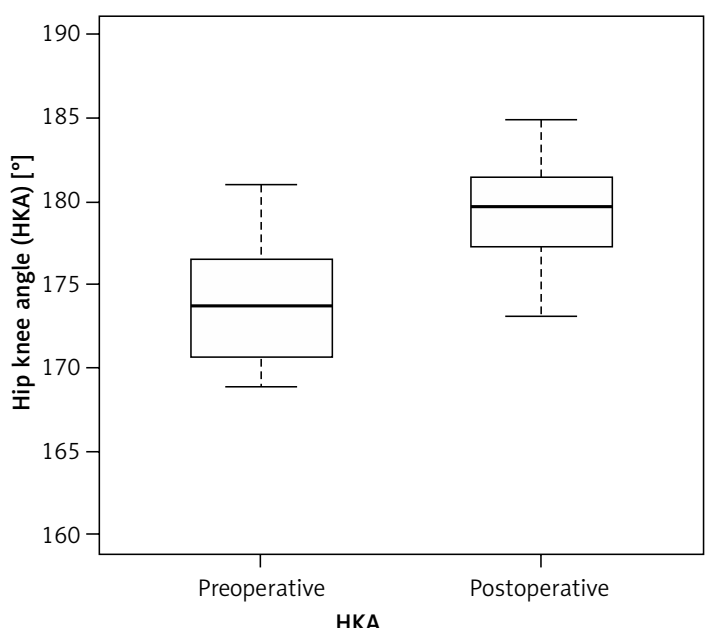

Figure 5. Box plot of the range of the hip-knee-ankle angle (HKA) preoperatively and postoperatively with illustration of the median angle (-), the area of $25-75 \%$ of the cases ( $\square$ ), as well as the maximum $(T)$ and minimum scores $(\perp)$ 


\section{Discussion}

The numbers of knee replacement procedures is steadily rising due to the changing demographic profile, with an increasing life expectancy in highly developed countries as well as a high prevalence of knee OA in the elderly population [21]. There is a growing interest in the field to develop patient-specific treatment modalities [22] to adequately address every particular stage of the disease and the individual requirements of each patient. Within this context, bi-compartmental knee replacements have been developed to preserve the intact compartment and the anterior cruciate ligament, which would otherwise be sacrificed in TKA approaches [8, 11, 12]. However, previous studies analyzing the outcome of monolithic, off-the-shelf bi-compartmental arthroplasty have demonstrated poor results [8] or high revision rates, with up to $18 \%$ conversions to TKA [9]. To our knowledge, this is the first study to analyze the precision of implant positioning and frontal plane alignment after customized bi-compartmental knee arthroplasty using both patient-specific instruments and implants (iDuo G2; ConforMIS Inc.).

Our study revealed that the treatment of our patients with the described customized BKA system can result in a nearly physiological frontal plane alignment (Figure 4). This indicates that the iDuo system is able to re-establish a near-physiological mechanical knee axis to an extent that is comparable to UKA systems using conventional off-the-shelf or custom-made techniques [12, 14-16]. Like several UKA systems, this BKA system is designed to resurface the diseased joint compartment. Therefore, the re-establishment of the physiological mechanical axis can be attributed to the restoration of the anatomical joint line to its pre-arthritic position (Figure 4). In patients with medial UKA the best results have been described when the postoperative mechanical axis is positioned over or slightly medial to the center of the knee, as overcorrection as well as excessive under-correction has been associated with early failure [23-26]. It has been shown that roughly $25 \%$ of patients treated with the Oxford UKR do have a residual varus alignment, without compromising the clinical or radiological outcome [27]. This supports the assumption that a slight under-correction might not be detrimental for partial knee replacements. In contrast, overcorrection has been associated with poorer outcomes in UKA, as it might promote development of OA in the unresurfaced contralateral compartment $[27,28]$. This may also apply for the custom-made BKA system described in this article. Naturally, the resurfacing concept in partial knee replacement such as UKA or BKA is different from TKA systems that are de- signed to correct toward neutral mechanical leg alignment via bony cuts that are positioned $90^{\circ}$ to the mechanical axes.

As the level of deformity correction is dependent on the gap-balancing step that is included in the iDuo system, we attribute the achieved levels of axis correction to this step along with the subsequent choice of the insert height. Thus, even higher levels of correction are possible but might result in overstuffing of the compartment and overcorrection, which should be avoided in UKA as outlined above $[27,28]$. As custom-made BKA approaches also preserve all knee ligaments, such as UKA, and do not alter the knee structure like TKA, we believe that the observed residual varus deformity will result in beneficial outcomes. The initial clinical data with custom-made BKA are promising and support this conclusion [11]. Nevertheless, it remains to be seen whether any persisting varus deformity after custom-made BKA will lead to equally good mid- and long-term results as after UKA.

Our results can be compared to a previous study investigating radiological outcomes following implantation of an off-the-shelf BKA system (Journey Deuce; Smith \& Nephew, Inc.) [29]. In this study, 137 patients who received a Journey Deuce implant for anteromedial OA were enrolled. More than $90 \%$ of these patients had a central knee alignment postoperatively [29]. In contrast to our data, the study demonstrated an overcorrection in $3.6 \%$ of the cases (5 patients) postoperatively [29], which we observed in one patient only (3.3\%) with an HKA of $184.9^{\circ}$. This is most likely due to the awareness and gap-balancing step used to avoid overcorrection of the mechanical axis, as already mentioned. Therefore, both custom-made and offthe-shelf BKA systems can facilitate the re-establishment of a central mechanical knee axis [30].

Our study is limited to the presentation of radiographic data of the iDuo system. To date, only preliminary outcome data on this custom-made BKA are available in the literature, but they are very promising so far [11]. The existing clinical data on off-the-shelf BKA using modular or mono-block implant systems demonstrate inferior overall survival of BKA compared to TKA $[8,9]$. Shah et al. observed a significantly greater range of motion in BKA patients compared to TKA, but nearly similar clinical and functional scores between both approaches at 24-month follow-up [31]. Morrison et al. reported a markedly higher short-term complication rate in BKA (28.6\%) compared to TKA (6.1\%) [32]. Palumbo et al. reported that after implantation of an off-the-shelf BKA system, $14 \%$ had to be converted into a TKA after 21 months of follow-up [33]. Using the same system, Müller et al. obtained an $82 \%$ survival rate (35 patients) after 2 years, with 18\% (8 patients) 
being converted to TKA due to pain, malalignment or instability [9]. Considering the unsatisfactory clinical outcomes of the published off-the-shelf BKA systems to date, further long-term clinical evaluation of the iDuo system is mandatory. Initial clinical short-term experiences are promising $[11,12]$, and a clinical long-term multicenter study using this device is currently ongoing. Apart from providing a possibility to re-establish normal knee axis alignment, the patient-specific BKA system described here can be used to provide an optimized fit of the implants with only minimal overhang or subsidence (less than $2 \mathrm{~mm}$ in all cases). It remains to be seen whether BKA using patient-specific implants and instruments will facilitate optimized clinical outcomes and results compared to standard BKA or TKA systems with respect to knee function, pain and quality of life.

Our radiographic analyses have shown that the iDuo implant system is able to precisely cover the tibial cortex without overhang or underhang in both planes within $2 \mathrm{~mm}$ accuracy margins, which corresponded well with what was seen in custom-made UKA using similar technologies [14-16]. This is in contrast to many off-the-shelf UKA systems that reveal insufficient cortical bone coverage due to their implant design and a consequently higher risk of implant subsidence and failure over time [23-25, 34, 35].

Moreover, the described customized BKA system has been shown to accurately restore the patient's individual tibial slope, with a mean preoperative tibial slope of $6.38 \pm 2.4^{\circ}$ and postoperative tibial slope of $5.77 \pm 1.97^{\circ}$. Several studies have underlined the importance of tibial slope restoration in UKA in order to approximate natural knee kinematics postoperatively while avoiding tibial component loosening [36, 37].

The accuracy of femoral component positioning in this patient-specific BKA system has also been confirmed by the conformity of the measured extent of posterior femoral condyle resection with the preoperative iView plan. Furthermore, the anatomic femoral design is intended to avoid edge-loading and, in combination with the restoration of the individual tibial slope, can result in the restoration of the physiologic patient-specific knee kinematics [10, 12]. Similar levels of accuracy were seen when a patient-specific UKA system was used in medial knee OA [14-16].

Patients undergoing knee resurfacing with this novel patient-specific platform have to be informed about the radiation they are exposed to in order to acquire the CT data set needed for production of the prosthesis. After CT imaging is performed, it takes approximately 8 weeks until the prostheses and the cutting guides are delivered in a sterilized form [11].
In conclusion, this study illustrates that this CTbased fixed bearing customized bi-compartmental knee resurfacing system enables the surgeon to restore the arthritic joint surface, and facilitates a nearly perfect fit and positioning of the implant components with full coverage of tibial cortex. These advantages might result in improved clinical results. At present this is the only radiographic study on this novel patient-customized bi-compartmental knee replacement system with promising data thus far. However, further follow-up studies including long-term clinical outcome parameters are mandatory to further clarify whether this system constitutes a valuable alternative treatment modality for patients with bi-compartmental knee OA.

\section{Acknowledgments}

The authors thank Mrs. Fritzsche for her help collecting the radiographic data.

This publication was supported by the Open Access Publication Fund of the University of Wuerzburg.

\section{Conflict of interest}

No benefits in any form have been received or will be received from a commercial party related, directly or indirectly, to the subject of this article. Moreover, the authors have full control of all primary data and will agree to allow the journal to review the data if requested. AFS and AR are teaching consultants for ConforMIS Inc. MR received institutional support for training activities.

\section{References}

1. Heekin RD, Fokin AA. Incidence of bicompartmental osteoarthritis in patients undergoing total and unicompartmental knee arthroplasty: is the time ripe for a less radical treatment? J Knee Surg 2014; 27: 77-81.

2. Yamabe E, Ueno T, Miyagi R, Watanabe A, Guenzi C, Yoshioka $\mathrm{H}$. Study of surgical indication for knee arthroplasty by cartilage analysis in three compartments using data from Osteoarthritis Initiative (OAI). BMC Musculoskelet Disord 2013; 14: 194.

3. Nam D, Nunley RM, Barrack RL. Patient dissatisfaction following total knee replacement: a growing concern? Bone Joint J 2014; 96-B (11 Suppl. A): 96-100.

4. Bourne RB, Chesworth BM, Davis AM, Mahomed NN, Charron KD. Patient satisfaction after total knee arthroplasty: who is satisfied and who is not? Clin Orthop Relat Res 2010; 468: 57-63.

5. Von Keudell A, Sodha S, Collins J, Minas T, Fitz W, Gomoll AH. Patient satisfaction after primary total and unicompartmental knee arthroplasty: an age-dependent analysis. Knee 2014; 21: 180-4.

6. Isaac SM, Barker KL, Danial IN, Beard DJ, Dodd CA, Murray $D W$. Does arthroplasty type influence knee joint proprioception? A longitudinal prospective study comparing total and unicompartmental arthroplasty. Knee 2007; 14: 212-7. 
7. Thienpont E, Schwab PE, Fennema P. A systematic review and meta-analysis of patient-specific instrumentation for improving alignment of the components in total knee replacement. Bone Joint J 2014; 96-B: 1052-61.

8. Tria AJ Jr. Bicompartmental knee arthroplasty: the clinical outcomes. Orthop Clin North Am 2013; 44: 281-6.

9. Müller M, Matziolis G, Falk R, Hommel H. [The bicompartmental knee joint prosthesis Journey Deuce: failure analysis and optimization strategies]. Orthopade 2012; 41: 894-904.

10. Steinert AF, Sefrin L, Hoberg M, Arnholdt J, Rudert M. [Individualized total knee arthroplasty]. Orthopade 2015; 44: 290-2, 4-301.

11. Steinert AF, Beckmann J, Holzapfel BM, Rudert M, Arnholdt J. Bicompartmental individualized knee replacement: use of patient-specific implants and instruments (iDuo). Oper Orthop Traumatol 2017; 29: 51-8.

12. Beckmann J, Steinert A, Zilkens C, et al. [Partial replacement of the knee joint with patient-specific instruments and implants (ConforMIS iUni, iDuo)]. Orthopade 2016; 45: 322-30.

13. Fitz W. Unicompartmental knee arthroplasty with use of novel patient-specific resurfacing implants and personalized jigs. J Bone Joint Surg Am 2009; 91 Suppl 1: 69-76.

14. Koeck FX, Beckmann J, Luring C, Rath B, Grifka J, Basad E. Evaluation of implant position and knee alignment after patient-specific unicompartmental knee arthroplasty. Knee 2011; 18: 294-9.

15. Arnholdt J, Holzapfel BM, Sefrin L, Rudert M, Beckmann J, Steinert AF. [Individualized unicondylar knee replacement: use of patient-specific implants and instruments]. Oper Orthop Traumatol 2017; 29: 31-9.

16. Demange MK, Von Keudell A, Probst C, Yoshioka $H$, Gomoll AH. Patient-specific implants for lateral unicompartmental knee arthroplasty. Int Orthop 2015; 39: 1519-26.

17. Gudena R, Pilambaraei MA, Werle J, Shrive NG, Frank CB. A safe overhang limit for unicompartmental knee arthroplasties based on medial collateral ligament strains: an in vitro study. J Arthroplasty 2013; 28: 227-33.

18. Pfitzner T, Perka C, Matziolis G. [Patella height after total knee replacement: influence of the radiological setting]. Orthopade 2009; 38: 616-21.

19. Springorum HR, Baier C, Craiovan B, et al. [Patella nav igation in computer-assisted TKA: intraoperative measurement of patellar kinematics. Video article]. Orthopade 2016; 45: 569-72.

20. Heesterbeek PJ, Beumers MP, Jacobs WC, Havinga ME, Wymenga $A B$. A comparison of reproducibility of measurement techniques for patella position on axial radiographs after total knee arthroplasty. Knee 2007; 14: 411-6.

21. Nemes S, Rolfson O, W-Dahl A, et al. Historical view and future demand for knee arthroplasty in Sweden. Acta Orthop 2015; 86: 426-31.

22. Fu H, Wang J, Zhou S, et al. No difference in mechanical alignment and femoral component placement between patient-specific instrumentation and conventional instrumentation in TKA. Knee Surg Sports Traumatol Arthrosc 2015; 23: 3288-95.

23. Emerson RH Jr, Higgins LL. Unicompartmental knee arthroplasty with the oxford prosthesis in patients with medial compartment arthritis. J Bone Joint Surgery Am 2008; 90: 118-22.

24. Kennedy WR, White RP. Unicompartmental arthroplasty of the knee. Postoperative alignment and its influence on overall results. Clin Orthop Relat Res 1987; 221: 278-85.

25. Ansari S, Newman JH, Ackroyd CE St. Georg sledge for medial compartment knee replacement. 461 arthroplasties followed for 4 (1-17) years. Acta Orthop Scand 1997; 68: 430-4.

26. Gulati A, Pandit H, Jenkins C, Chau R, Dodd CA, Murray $D W$. The effect of leg alignment on the outcome of unicompartmental knee replacement. J Bone Joint Surgery $\mathrm{Br}$ 2009; 91: 469-74

27. Emerson RH Jr. Preoperative and postoperative limb alignment after Oxford unicompartmental knee arthroplasty. Orthopedics 2007; 30 (5 Suppl): 32-4.

28. Deshmukh RV, Scott RD. Unicompartmental knee arthroplasty: long-term results. Clin Orthop Relat Res 2001; 392: 272-8.

29. Rolston L, Siewert K. Assessment of knee alignment after bicompartmental knee arthroplasty. J Arthroplasty 2009; 24: 1111-4.

30. Sharma L, Song J, Felson DT, Cahue S, Shamiyeh E, Dunlop DD. The role of knee alignment in disease progression and functional decline in knee osteoarthritis. JAMA 2001; 286: 188-95.

31. Shah SM, Dutton AQ, Liang S, Dasde S. Bicompartmental versus total knee arthroplasty for medio-patellofemoral osteoarthritis: a comparison of early clinical and functional outcomes. J Knee Surg 2013; 26: 411-6.

32. Morrison TA, Nyce JD, Macaulay WB, Geller JA. Early adverse results with bicompartmental knee arthroplasty: a prospective cohort comparison to total knee arthroplasty. J Arthroplasty 2011; 26 (6 Suppl): 35-9.

33. Palumbo BT, Henderson ER, Edwards PK, Burris RB, Gutierrez S, Raterman SJ. Initial experience of the Journey-Deuce bicompartmental knee prosthesis: a review of 36 cases. J Arthroplasty 2011; 26 (6 Suppl): 40-5.

34. Carpenter DP, Holmberg RR, Quartulli MJ, Barnes CL. Tibial plateau coverage in UKA: a comparison of patient specific and off-the-shelf implants. J Arthroplasty 2014; 29: 1694-8.

35. Fitzpatrick C, FitzPatrick D, Lee J, Auger D. Statistical design of unicompartmental tibial implants and comparison with current devices. Knee 2007; 14: 138-44.

36. Servien E, Saffarini M, Lustig S, Chomel S, Neyret P. Lateral versus medial tibial plateau: morphometric analysis and adaptability with current tibial component design. Knee Surg Sports Traumatol Arthrosc 2008; 16: 1141-5.

37. Hernigou P, Deschamps G. Posterior slope of the tibial implant and the outcome of unicompartmental knee arthroplasty. J Bone Joint Surg Am 2004; 86-A: 506-11. 